\title{
NUEVOS REGISTROS DE MURCIÉLAGOS PARA EL ESTADO DE NAYARIT, MÉXICO
}

\author{
JOAQUÍN ARROYO-CABRALES ${ }^{1}$, OSCAR J. POLACO ${ }^{1}$, DON E. WILSON ${ }^{2}$ Y \\ ALFREDL.GARDNER ${ }^{3}$ \\ ${ }^{1}$ Laboratorio de Arqueozoología "M. en C. Ticul Álvarez Solórzano", Subdirección de \\ Laboratorios y Apoyo Académico, INAH, Moneda \# 16, Col. Centro, 06060 México, D. F. \\ ${ }^{2}$ Division of Mammals, National Museum of Natural History, The Smithsonian Institution, \\ Washington, D. C. 20560, EUA \\ ${ }^{3}$ USGS Patuxent Wildlife Research Center, PO Box 37012, National Museum of Natural \\ History, Room 378 MRC 111, Washington, DC 20013-7012, EUA \\ correoelectrónico: arromatu5@yahoo.com.mx
}

RESUMEN: Basados en el estudio de ejemplares depositados en museos y colectas de campo recientes, se documenta la presencia de 19 especies de murciélagos (Mammalia: Chiroptera) para el estado de Nayarit, en el centro occidente de México. Las especies registradas pertenecen a las familias Phyllostomidae (3), Vespertilionidae (11) y Molossidae (5), de ellas once son nuevos registros para el estado. Dichas especies, sumadas a las 47 previamente conocidas, ubican a Nayarit como uno de los estados más diversos del país con el 42\% de la biodiversidad chiropterofaunística.

PALABRAS CLAVE: Chiroptera, México, Nayarit, nuevos registros.

ABSTRACT: Based on the study of museum specimens and results from recent field collections, 19 species of bats (Mammalia, Chiroptera) are newly recorded for the state of Nayarit in west-central México. Eleven of these are new records for the state. Those species pertain to the bat families Phyllostomidae (3), Vespertilionidae (11), and Molossidae (5). Those new records, along with the previous 47 known species, give the state $42 \%$ of the bat diversity of México, making it one of the most diverse states in the country.

KEY WORDS: Chiroptera, México, Nayarit, new records.

\section{INTRODUCCIÓN}

El estado de Nayarit se localiza a lo largo de la costa del centro-oeste de México, entre los $21^{\circ}$ y los $23^{\circ}$ de latitud norte. Su compleja topografía incluye amplias extensiones de planicie costera, así como la región al poniente de la Sierra Madre Occidental, donde las escarpas y los picos exceden los 2,000 metros de altitud (Figura 1). Con este pronunciado relieve y sus exposiciones costeras, Nayarit posee una variedad de ambientes y de zonas bióticas que lo hace uno de los estados con la mayor diversidad de vegetación nativa (Flores Villela y Gerez, 1994). 
Siguiendo la clasificación de zonas de vida propuesta por Goldman (1951), Nayarit contiene elementos del Trópico Inferior, las subdivisiones árida y húmeda del Trópico Superior, las Australes Inferior y Superior y las Zonas de Transición. A lo largo de los valles lacustres, las comunidades de plantas típicas de las elevaciones bajas interiores se distribuyen en las montañas del estado e interdigitan con la vegetación de altura de una manera complicada. Debido a su compleja fisiografía, Nayarit ocupa una importante posición para comprender los modelos de distribución y dispersión de los mamíferos y para la resolución de problemas taxonómicos en este grupo en el oeste de México, especialmente cierto en el caso de los pequeños mamíferos; a pesar de esto, se han realizado pocos estudios completos de la fauna del estado.

Las colecciones depositadas en los museos representan el conocimiento acumulado a través del tiempo, cada museo guarda una parte de dicho conocimiento y, ninguno duplica exactamente los materiales de otro (Mehrhoff, 1996). A ello hay que agregar la importancia de documentar la presencia de una especie en un área dada, ya que son los datos requeridos en los estudios biogeográficos y de otro tipo.

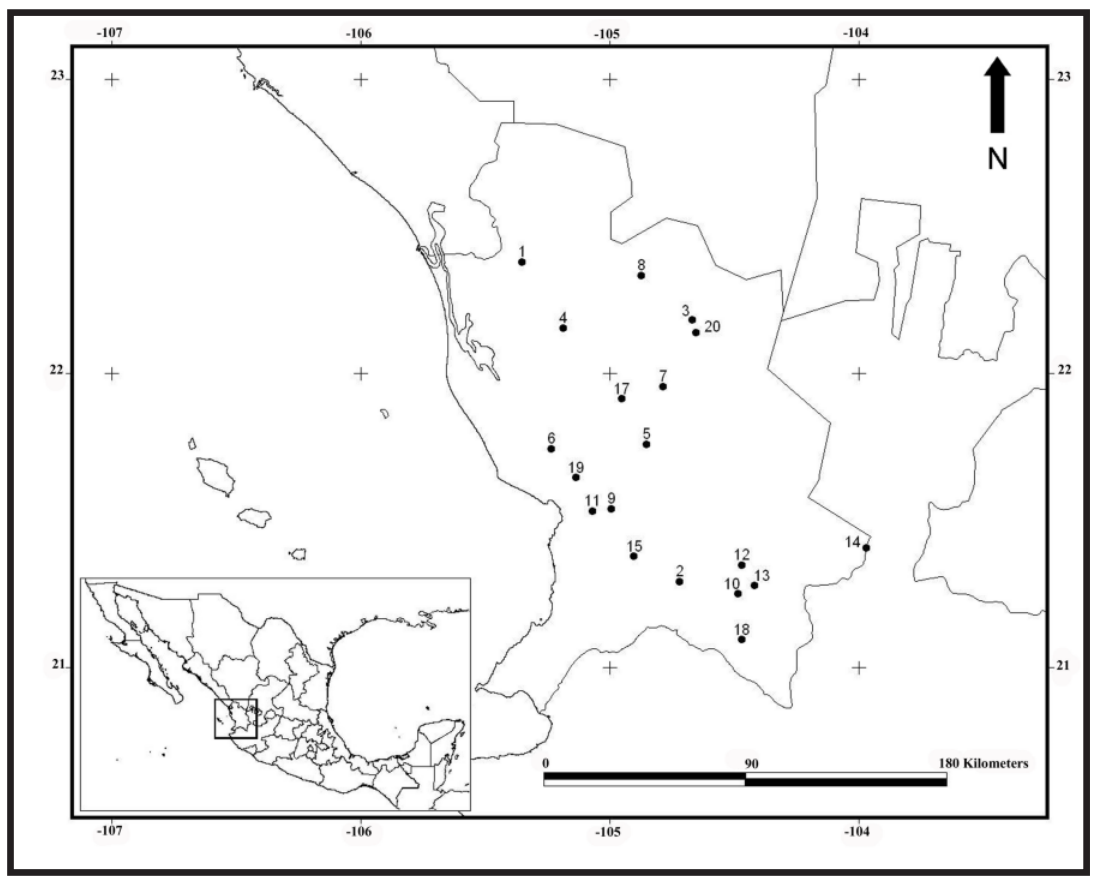

Figura 1. Mapa de Nayarit donde se señalan las localidades de colecta de los murciélagos estudiados. Los números corresponden a las localidades proporcionadas en el Cuadro 2. 
En Nayarit, se ha registrado la presencia de 47 especies de murciélagos de siete familias (Cuadro 1, modificado de Ceballos y Oliva, 2005). Tres de ellas tienen su límite de distribución más norteño en el estado: Diclidurus albus, Glyphonycteris sylvestris y Nyctinomops laticaudatus.

Como parte del proyecto de estudio de los mamíferos del estado de Nayarit que se encuentran alojados en la colección de la División de Mamíferos del National Museum of Natural History, Washington, D. C. (Smithsonian Institution), se examinaron los ejemplares pertenecientes a siete familias de murciélagos; en este reporte se excluyó a la familia Phyllostomidae por el gran número de ejemplares de cada especie, exceptuando a Glyphonycteris sylvestris, Choeronycteris mexicana y Centurio senex y se incluyó a la famillia Vespertilionidae y Molossidae. El estudio se complementa con ejemplares colectados en años recientes por estudiantes de la carrera de Biología de la Escuela Nacional de Ciencias Biológicas del Instituto Politécnico Nacional, México durante sus prácticas de campo en la localidad de La Noria (coordenadas gps $21^{\circ} 29^{\prime} 7^{\prime \prime} \mathrm{N}, 104^{\circ} 59^{\prime} 39^{\prime \prime}$ O). Se incorporan algunas especies cuya presencia ya había sido documentada en la literatura, pero de las que no se proporcionaron las medidas de los ejemplares, ni otros datos pertinentes. Cabe mencionar que todos los ejemplares que se hallan depositados en el Smithsonian Institution fueron enlistados por López-Wilchis y López Jardines (1998, 1999, 2000), el único dato proporcionado fue su procedencia; dicha información fue retomada en algunas de las monografías que componen el trabajo de Ceballos y Oliva (2005).

\section{MATERIAL YMÉTODOS}

Los ejemplares se identificaron usando las claves de Hall (1981), Alvarez et al. (1994) y Medellín et al. (2009), así como la bibliografía pertinente a cada caso. Las medidas somáticas proceden de las etiquetas de cada ejemplar; las craneales se tomaron con un vernier digital Ultra-Cal II hasta décimas de milímetro, siguiendo a Handley (1959) y Owen (1987), excepto en la longitud total del cráneo que en este caso se tomó incluyendo a los incisivos. El promedio, la mínima y la máxima, o las medidas de uno a tres ejemplares, se proporcionan en el Apéndice. Las abreviaturas usadas en el mismo son: $\mathrm{N}$ = número de ejemplares; TOTA = longitud total del cuerpo; COLA = longitud de la cola; PATA = longitud de la pata trasera derecha; OREJA = longitud de la oreja en fresco; $\mathrm{AB}=$ longitud del antebrazo; TRA = longitud del tragus; GLS = longitud mayor del cráneo; COBA = longitud desde el extremo anterior de la premaxila hasta la proyección posterior de los cóndilos occipitales; ZYGO = anchura cigomática; MAST = anchura mastoidea; ORBI = anchura postorbital; $\mathrm{BRAB}$ = anchura de la caja craneal; BRAH = altura del cráneo; PALA = longitud del paladar; POST = distancia del borde posterior del paladar al borde anterior del foramen magnum; MAXIL = longitud de la hilera de dientes molariformes maxilares; WM2 = anchura entre la parte anterior bucal 
Cuadro 1. Listado de las especies de murciélagos que se conocen para el estado de Nayarit (modificado de Ceballos y Oliva, 2005).

ESPECIE Ceballos y Oliva, 2005 Este trabajo

Balantiopteryx plicata

Diclidurus albus

$\mathrm{X}$

Noctilio leporinus

$\mathrm{X}$

Mormoops megalophylla

Pteronotus davyi

Pteronotus parnellii

Pteronotus personatus

Macrotus waterhousii

Glyphonycteris sylvestris

Desmodus rotundus

Choeronycteris mexicana

Glossophaga commissarisi

Glossophaga soricina

Leptonycteris yerbanuenae

$\mathrm{X}$

$\mathrm{X}$

$\mathrm{X}$

$\mathrm{X}$

$\mathrm{X}$

$\mathrm{X}$

$\mathrm{X}$

X

$\mathrm{X}$

Leptonycteris nivalis

Anoura geoffroyi

Hylonycteris underwoodi

Choeroniscus godmani

Sturnira lilium

Sturnira ludovici

Chiroderma salvini

Artibeus hirsutus

Artibeus jamaicensis

Artibeus intermedius

Dermanura azteca

Dermanura phaeotis

Dermanura tolteca

Centurio senex

Natalus stramineus

Antrozous pallidus

Bauerus dubiaquercus

$\mathrm{X}$

$\mathrm{X}$

$\mathrm{X}$

$\mathrm{X}$

$\mathrm{X}$

$\mathrm{X}$

$\mathrm{X}$

$\mathrm{X}$

$\mathrm{X}$

$\mathrm{X}$

$\mathrm{X}$

$\mathrm{X}$

$\mathrm{X}$

$\mathrm{X}$

$\mathrm{X}$

$\mathrm{X}$

$\mathrm{X}$

$\mathrm{X}$

$\mathrm{X}$

$\mathrm{X}$

X

Coryhorhinus townsendii

$\mathrm{X}$

Eptesicus furinalis

Eptesicus fuscus *

Lasiurus blossevillii

Lasiurus cinereus

$X$

X

Lasiurus intermedius

$\mathrm{X}$

$\mathrm{X}$

Lasiurus xanthinus

$\mathrm{X}$ 
Cuadro 1. Continuación...

\begin{tabular}{lcc}
\hline ESPECIE & Ceballos & \\
\hline Myotis auriculus & & Este trabajo \\
Myotis californicus & $\mathrm{X}$ & $\mathrm{X}$ \\
Myotis carteri & $\mathrm{X}$ & \\
Myotis ciliolabrum & $\mathrm{X}$ & \\
Myotis fortidens & $\mathrm{X}$ & \\
Myotis findleyi & $\mathrm{X}$ & \\
Myotis thysanodes & & $\mathrm{X}$ \\
Myotis velifer & $\mathrm{X}$ & $\mathrm{X}$ \\
Myotis yumanensis & $\mathrm{X}$ & $\mathrm{X}$ \\
Pipistrellus hesperus & & $\mathrm{X}$ \\
Rhogeessa gracilis & & $\mathrm{X}$ \\
Rhogeessa parvula & $\mathrm{X}$ & \\
Cynomops mexicanus & $\mathrm{X}$ & $\mathrm{X}$ \\
Eumops perotis & & $\mathrm{X}$ \\
Molossus aztecus & & $\mathrm{X}$ \\
Molossus rufus & $\mathrm{X}$ & $\mathrm{X}$ \\
Molossus sinaloae & & $\mathrm{X}$ \\
Nyctinomops aurispinosus & $\mathrm{X}$ & \\
Nyctinomops femorosaccus & & $\mathrm{X}$ \\
Nyctinomops laticaudatus & & \\
TOTAL & 47 & \\
\hline
\end{tabular}

de los M2 y DENT = longitud del dentario; TOOT = longitud de la hilera de dientes molariformes mandibulares (Apéndice I).

En el tratado siguiente, se indica el nombre científico de la especie o subespecie y el autor y año de la descripción, las localidades de colecta (las que se registran tal y como aparecen en las etiquetas, ver Apéndice 2, Figura 1), el número de ejemplares (entre paréntesis), la distribución conocida de la especie o subespecie (generalmente de acuerdo a Jones et al., 1988) y comentarios complemento de cada uno de los taxa estudiados. Se sigue la nomenclatura propuesta por Ceballos et al. (2005).

Los ejemplares están depositados en las colecciones de mamíferos del $\mathrm{Na}$ tional Museum of Natural History (USNM), del The University of Kansas Museum of Natural History (UK) (Apéndice 3) y, la colección osteológica de referencia del Laboratorio de Arqueozoología "M. en C. Ticul Álvarez Solórzano" de la Subdirección de Laboratorios y Apoyo Académico del Instituto Nacional de Antropología e Historia (DP). Para esta última no se proporcionan los números de catálogo debido a que aún se hallan en el proceso de catalogación. 


\section{TRATADOTAXONÓMICO}

Se documenta la presencia de 19 especies de murciélagos pertenecientes a tres familias: Phyllostomidae (3), Vespertilionidae (11) y Molossidae (5).

Familia Phyllostomidae

\section{Glyphonycteris sylvestris Thomas, 1896}

Ejemplares examinados 2: El Venado, 2 mi E of, 2-Mar-1964 (1); La Noria (1).

Esta especie se localiza en la vertiente del Pacífico, desde Nayarit hacia el sur y en el Golfo desde Veracruz hasta el sur de Brasil. El ejemplar hembra proveniente de El Venado fue registrado previamente por Jones (1964). El procedente de La Noria, una hembra sin señales de actividad reproductiva, se recolectó en noviembre de 1998; adicionalmente se le extrajeron los artrópodos asociados, los que están bajo estudio (M. Vargas, comunicación personal, 2008).

\section{Choeronycteris mexicana Tschudi, 1844}

Ejemplares examinados 6: Acaponeta, 300 ft, 2-Jul-1897 (1); San Pedro Lagunillas, 2 mi E, E side lake, 18-Mar-1975 (1); Arroyo Taberna, 2 mi NW Mesa del Nayar, 4900 ft, 22Ene-1975 (1); Arroyo Taberna, 2 mi WNW Mesa del Nayar, 4900 ft, 23-Ene-1975 (3). Este murciélago polinívoro se distribuye en la mayor parte del territorio nacional, así como en una franja del suroeste de EUA, hacia el sur alcanza Honduras. Esta especie fue registrada de manera errónea para las Islas Marías, Wilson (1991) demostró que el ejemplar documentado es un Glossophaga soricina mutica. Por ello, los ejemplares aquí estudiados constituyen el primer registro real de la especie en el estado.

\section{Centurio senex senex Gray, 1842}

Ejemplares examinados 28: Teponahuaxtla, 26-Feb-1975(1); El Casco, $1.2 \mathrm{mi} \mathrm{S}$, by road, Rio Chilte, 480 ft, 16-18-Oct-1975 (24); Villa Hidalgo, 4.8 mi W, 4-Jun-1984 (1); San Miguel del Zapote, 8 mi NE Mesa del Nayar, 5-Jun-1984 (2). Esta especie se distribuye por las vertientes costeras de México, desde Sinaloa y Tamaulipas hasta Centro y Sudamérica. Dos registros previos proceden, uno del pueblo de Palapita (León y Romo, 1991) en el sur del estado y el otro de Acaponeta (Elizalde-Arellano et al., 2004) en el norte; las localidades que aquí se consignan son de la parte central, lo que complementa el conocimiento de la distribución de la especie, cuyo límite noroccidental está en el norte de Sinaloa (Jones et al., 1972).

De los ejemplares estudiados, dos hembras colectadas a principios de junio tenían un embrión cada una que medían 26 y 25 mm, de longitud corona-abdomen 
(crown-rump) respectivamente, mientras que tres machos colectados en octubre tuvieron los testículos escrotados. Un ejemplar hembra (TAMU 40907) colectado a 0.5 mi S Teponahuaxtla, en julio de 1981, aparece en un listado de las especies de mamíferos del estado de Nayarit que se encuentran bajo resguardo de la Texas Wildlife \& Cooperative Collection, Texas A\&M University, en College Station, Texas, EUA (TAMU).

Familia Vespertilionidae

\section{Antrozous pallidus packardi Martin y Schmidly, 1982}

Ejemplares examinados 2: Arroyo Taberna, 2 mi WNW Mesa del Nayar, 4900 ft, 22-Oct1975 (1); Santa Teresa, 13 km SW Rancho Viejo, 20-Ene-1977 (1). Esta especie se encuentra desde el centro de México hasta Estados Unidos de América. La subespecie se distribuye siguiendo la vertiente poniente de la Sierra Madre Occidental, desde Jalisco hasta el sur de Sonora. Martin y Schmidly (1982) revisaron estos ejemplares, los que son parte de la serie típica de la subespecie. Un macho colectado en el mes de octubre presentaba los testículos escrotados.

\section{Bauerus dubiaquercus (Van Gelder, 1959)}

Ejemplares examinados 1: La Noria, 4-Dic-1994. Esta especie está bien representada por 46 ejemplares procedentes de las Islas Tres Marías, así como por registros aislados en el continente, que van desde Jalisco hasta Costa Rica (Wilson, 1991), incluyendo la Península de Yucatán (Juárez et al., 1988). El ejemplar estudiado, un macho con medida testicular de $5 \mathrm{~mm}$, representa el primer registro de la especie en las tierras continentales de Nayarit. Las medidas craneales quedan comprendidas en el intervalo de las medidas proporcionadas para los machos procedentes de las Islas Tres Marías por Engstrom y Wilson (1981), de hecho en la mayoría de ellas son muy cercanas a la media de las mismas. Sin embargo, las medidas somáticas son diferentes del promedio, una posible explicación a esto es la enorme variación encontrada en el registro de estas medidas por los preparadores, aspecto demostrado por Santos Moreno (1994).

\section{Corynorhinus townsendii australis (Handley, 1955)}

Ejemplares examinados 1: Coapan, $1.8 \mathrm{mi} \mathrm{NW}$, by road, $4650 \mathrm{ft}, 11-F e b-1975$. Esta subespecie se distribuye en la mayor parte de México, con excepción del noroeste. La designación genérica sigue lo señalado por Ramírez-Pulido et al. (1996). Este ejemplar coincide en tamaño con lo señalado para la subespecie por Tumlison (1991), puesto 
que la medida del antebrazo (42.7) es semejante al promedio dado para C. t. australis (42.8) y mayor que el de las hembras de C.t. pallescens de México (40.2), confirmando así lo propuesto por Bogan (1999) para los ejemplares que fueran colectados en el estado.

\section{Eptesicus furinalis gaumeri (J. A. Allen, 1897)}

Ejemplares examinados 2: Jalcocotán, 2 mi E, 17-Ene-1977 (1); La Noria, 4-Dic-1994(1). Esta subespecie se distribuye en ambas vertientes de México, desde Jalisco, San Luis Potosí y Tamaulipas hasta el norte de Centroamérica. Las medidas de los ejemplares quedan dentro del intervalo de la subespecie proporcionadas por Davis (1965). Los ejemplares nayaritas constituyen el registro más noroccidental de la especie, ya que los más norteños procedían del centro (Talpa de Allende, La Cuetas) y del sur (Cihuatlán, Pihuamo) del estado de Jalisco (Watkins et al., 1972).

R. A. Medellín (comunicación personal, 1998) considera que el ejemplar de Jalcocotán pertenece a la especie E. chiriquinus (sensu Gardner, 2008) por la forma de la región interorbital; dicha consideración se ve reflejada en el mapa de distribución de E. chiriquinus (= E. andinus) de Medellín et al. (2009); lo mismo lo presentan Ceballos y Oliva (2005) bajo la especie E. brasiliensis. Sin embargo, el ejemplar se identifica como $E$. furinalis debido tanto a su pelaje corto, como a que sus medidas quedan dentro de la variación de ejemplares colectados en Sudamérica y asignados a $E$. furinalis (Williams, 1978). Asimismo, los caracteres merísticos de los dos ejemplares los identifican como E. furinalis siguiendo la clave propuesta para el género por López-González (2005). Por otro lado, el carácter exclusivo para E. chiriquinus, el abultamiento supraorbital, formado por una depresión que va desde la abertura anterior del canal infraorbital a lo largo del rostro hasta la constricción postorbital (C. O. Handley, Jr., comunicación personal, 1999), no se presenta en los ejemplares de Nayarit, en los que la región supraorbital es plana.

\section{Eptesicus fuscus miradorensis (H. Allen, 1886)}

Ejemplares examinados 12: San Pedro Lagunillas, 2 mi E, E side lake, 18-Mar-1975 (1); Estanzuela, 21-Mar-1975 (1); Jala, 10 km N, 4900 ft, 29,30-Oct-1975 (2); Coapan, 1.8 mi NW, by road, 4650 ft, 2-Nov-1975 (2); La Noria, 3, 4-Dic-1994 (6). El murciélago pardo Eptesicus fuscus se distribuye en toda la República Mexicana. La subespecie que aquí se registra se conoce del centro-norte de México (Sinaloa, Durango, centro de Coahuila y Tamaulipas) hasta Sudamérica. Bogan (1999) usó el nombre de Eptesicus serotinus para los ejemplares asignados a E. fuscus en el noroeste de México, que incluiría aquellos que se distribuyeran en Nayarit. Este nombre se basó en una propuesta de Koopman (1989), la que fue descartada por él mismo con posterioridad 
(Koopman, 1993, 1994), por ello no parece justificable utilizar el nombre que corresponde a una especie europea, para los ejemplares mexicanos.

Burnett (1983) describe la variación geográfica de esta especie con base en muestras de las poblaciones que habitan Norteamérica, pero el estudio no fue concluyente respecto a los caracteres diagnósticos para cada subespecie. Sin embargo, la coloración parece ser importante para la separación de las mismas (Allen, 1933; Engels, 1936); Miller (1897) indica algunos caracteres craneales de utilidad para separar E. f. miradorensis de la subespecie típica, pero aclara que no son $100 \%$ confiables.

Los murciélagos estudiados se asignan a esta subespecie con base tanto en la distribución geográfica, como en su coloración dorsal café negruzca con el vientre canela obscuro. La coloración que se presenta en los ejemplares del centro y sur de México, entre ellos dos pieles procedentes de Tuxpango, Veracruz, sitio ubicado a 100 $\mathrm{km}$ al sur de la localidad típica de E. f. miradorensis, Mirador, Veracruz (USNM 6993, 6998). Un ejemplar macho (UK 135721) fue colectado en Tepic en julio de 1935. El mismo aparece en un listado de las especies de mamíferos del estado de Nayarit que se encuentran bajo resguardo del Museum of Natural History, The University of Kansas (UK).

\section{Myotis auriculus apache Hoffmeister y Krutzsch, 1955}

Ejemplares examinados 14: Estanzuela, 20, 22-Mar-1975 (6); Coapan, $1.8 \mathrm{mi} \mathrm{NW}$, by road, 4650 ft, 1, 2-Nov-1975 (5); La Noria, 3, 4-Dic-1994 (3). Esta subespecie se distribuye por el centro y oeste de México, desde Jalisco y Veracruz hacia el norte, sin ocupar el noroeste de la vertiente del Pacífico, excepto por dos registros previos, llegando a los EUA donde habita típicamente en los bosques de pino-encino (Bogan, 1999). Nuestros ejemplares coinciden con la coloración ocre grisáceo señalada para la subespecie por Alvarez y Polaco (1984). Al estudiar la historia y el estado taxonómico de M. auriculus, Genoways y Jones (1969) no realizan la revisión completa de los ejemplares asignados a dicha especie, lo que resultó en que proporcionan pocos caracteres diagnósticos para separar las subespecies, la excepción es la coloración pálida de $M$. a. apache en contraste con la de $M$. a. auriculus, carácter que sin embargo varia dentro de la misma población; por ello es recomendable que aquellos ejemplares procedentes de la zona de intergradación de ambas subespecies sean estudiados con mayor detenimiento.

\section{Myotis thysanodes thysanodes Miller, 1897}

Ejemplares examinados 8: Estanzuela, 20-Mar-1975 (1); Coapan, 1.8 mi NW, by road, 4650 ft, 1, 2-Nov-1975 (5); Rancho Sapotito, 3500 ft, 3, 4-Nov-1975 (2). Este murciélago 
se distribuye desde el sur de México (Chiapas y Oaxaca) hacia EUA. La subespecie típica ocurre en el centro y norte del país. En el noroeste de México, sólo se conocen registros aislados del norte de Baja California y Sonora (Bogan, 1999). Los ejemplares de esta especie se separan fácilmente de otras del género Myotis que se distribuyen en el Continente Americano, tanto por su mayor tamaño, como por la notoria franja de pelos largos que se presenta en el borde posterior del uropatagio (Hall, 1981).

\section{Myotis velifer velifer (J. A. Allen, 1890)}

Ejemplares examinados 1: El Refilion, 11-Mar-1975. La especie se conoce desde Centroamérica hasta EUA, asignándose a la subespecie típica aquellos ejemplares que se distribuyen desde el centro-norte de México hacia el sur. A diferencia de lo que ocurre en Sinaloa, donde es la especie de vespertiliónido más abundante (Jones et al., 1972), en Nayarit sólo se conocen dos ejemplares, ambos del sur del estado, uno procedente de Santa María del Oro (Polaco et al., 1992) y el que aquí se documenta.

\section{Myotis yumanensis lutosus Miller y G. M. Allen, 1928}

Ejemplares examinados 2: Cucharas, Rio Acaponeta, 22-Feb-1975(1); El Venado, 5 km E, 6-Jun-1984 (1). La especie se conoce desde el centro de México hacia el norte, por la Altiplanicie Mexicana y el noroeste del país hasta EUA. La subespecie M. y. lutosus ocupa la parte sureña de la distribución de la especie, desde San Luis Potosí, Zacatecas y el norte de Sinaloa hacia el sur. Los dos ejemplares son machos con testículos abdominales. El primer registro para el estado procede de Santa María del Oro (Polaco et al., 1992).

\section{Parastrellus hesperus hesperus (H. Allen, 1864)}

Ejemplares examinados 3: Arroyo Taberna, 2 mi WNW Mesa del Nayar, 4900 ft, 22Oct-1975 (2); San Miguel del Zapote, 8 mi NE Mesa del Nayar, 5-Jun-1984 (1). La subespecie se distribuye por el oeste de México, desde Morelos y Guerrero, hacia el norte hasta el oeste de EUA. El ejemplar de junio era una hembra lactante.

Rhogeessa gracilis Miller, 1897

Ejemplares examinados 1: Ahuacatlan, $8 \mathrm{mi}$ S, by road, 20-Ene-1977. Esta subespecie se distribuye por la Sierra Madre Occidental, desde Zacatecas hasta Oaxaca. Su presencia en Nayarit representa el registro más occidental para la especie. 
Familia Molossidae

\section{Eumops perotis californicus (Merriam, 1890)}

Ejemplares examinados 1: Teponahuaxtla, 24-Feb-1975. En los últimos 15 años, esta subespecie se ha registrado de varios estados del centro, oeste (Polaco et al., 1992) y del noroeste del país (Muñiz-Martínez et al., 2003), incluyendo a Nayarit, con el ejemplar que aquí se documenta. Su presencia en el estado corrobora lo continuo de su distribución en los estados de la vertiente del Pacífico desde Michoacán hasta Arizona, EUA (Hoffmeister, 1986). El ejemplar colectado es una hembra, la que presenta una anomalía dental, le falta el pequeño PM3 izquierdo.

\section{Molossus aztecus Saussure, 1860}

Ejemplares examinados 22: Estanzuela, $4500 \mathrm{ft}, 20$, 22-Mar-1975. Esta especie se distribuye en las tierras mésicas, arriba de $460 \mathrm{~m}$, de las porciones más norteñas de la Sierra Madre del Sur y la Cordillera Transvolcánica de México hacia el sur, a las tierra altas del centro de Nicaragua (Dolan, 1989). El registro de esta especie en Nayarit representa la ocurrencia más norteña, con excepción de un solo ejemplar conocido de Sinaloa (Gardner, 1966). Cuatro machos colectados en marzo presentaron los testículos escrotados. Otros seis tenían testículos abdominales, en tanto 12 hembras no mostraron evidencia de actividad reproductiva.

\section{Molossus sinaloae J. A. Allen, 1906}

Ejemplares examinados 8: El Refilion, 8, 11-Mar-1975. Como la especie precedente, se distribuye desde la porción más norteña de la Sierra Madre del Sur en Jalisco, hacia el sur y este hasta Morelos y la Península de Yucatán, llegando hasta Centro y Sudamérica (Dolan, 1989). Exceptuando al tipo de la especie que procede de Escuinapa, Sinaloa, no se conocían ejemplares más al norte de Teuchitlán, Jalisco, por lo que Dolan (1989) supuso que dicho ejemplar era un animal extraviado y no pertenecía a una población viable; el registro nayarita contradice este punto de vista. Se colectaron 5 hembras y 3 machos.

\section{Nyctinomops femorosaccus (Merriam, 1889)}

Ejemplares examinados 6: Teponahuaxtla, 24, 26-Feb-1975 (2); Arroyo Taberna, 2 mi WNW Mesa del Nayar, 4900 ft, 21, 22-Oct-1975, (2); San Blas, 18 km NE, El Palillo, 3Jun-1984 (1); San Miguel del Zapote, 8 mi NE, Mesa del Nayar, 5-Jun-1984 (1). Se distribuye en gran parte del oeste de México, desde el sur de Guerrero, incluyendo la 
Península de Baja California, hacia el noreste de México (Nuevo León) y sur de EUA. Se colectaron dos machos en el mes de junio, mientras que las capturas de febrero y octubre correspondieron a hembras.

\section{Nyctinomops laticaudatus ferrugineus (Goodwin, 1954)}

Ejemplares examinados 4: Teponahuaxtla, 24, 25-Feb-75. Se distribuye en las tierras tropicales de gran parte de México desde Tamaulipas (este) y Sinaloa (oeste) hacia el sur llegando a Sudamérica. Una hembra y tres ejemplares en los que no se identificó el sexo constituyen el registro.

\section{DISCUSIÓN}

Once de las 19 especies de murciélagos aquí documentadas representan nuevos registros para el estado de Nayarit, las que sumadas a las 47 previamente registradas, totalizan 58 especies, lo que representa el $42 \%$ de las 138 especies conocidas para México (Medellín et al., 2009). Lo anterior sin considerar a los ejemplares disponibles de la familia Phyllostomidae que, aunque ya conocidas la mayoría de las especies, pudieran representar algunas no colectadas en el estado. Recientemente Guerrero y Cervantes (2003) registraron la presencia de 70 especies de murciélagos en el estado de Jalisco, lo que era previsible de acuerdo a la hipótesis de que a mayor latitud, menor diversidad de murciélagos (Willig y Selcer, 1989), así en Nayarit se conocen sólo 14 especies de la Familia Vespertilionidae, en Jalisco son 22, coincidiendo con lo hallado por Patten (2004), un gradiente de murciélagos desde el Ártico hasta el Istmo de Panamá, donde la mayor diversidad de vespertiliónidos se presenta en el centro y oeste de México y el sur de EUA.

\section{CONCLUSIÓN}

Las 19 especies de murciélagos aquí documentadas, once de las cuales representan nuevos registros para el estado de Nayarit, sugieren la importancia que las colecciones y los inventarios tienen actualmente para completar el conocimiento de la fauna de un área y, eventualmente, procurar su conservación.

\section{AGRADECIMIENTOS}

El primer autor agradece a la Office of Fellowships and Grants, Smithsonian Institution, por el apoyo otorgado para la realización del estudio de los ejemplares que se hallan en el National Museum of Natural History, como parte de una estancia postdoctoral, al igual que al Instituto Nacional de Antropología e Historia por otorgarle 
una Beca de Capacitación para la realización de la estancia postdoctoral. Asimismo, agradecemos a los estudiantes de la Escuela Nacional de Ciencias Biológicas, IPN, por ceder los ejemplares recolectados a las colecciones del Instituto Nacional de Antropología e Historia. Finalmente, le damos las gracias al Biól. César A. Ríos Muñoz; Museo de Zoología, Facultad de Ciencias, UNAM, por su amabilidad en preparar el mapa que constituye la Figura 1 y a dos revisores anónimos que con sus comentarios mejoraron de manera significativa el trabajo.

\section{LITERATURACITADA}

Allen, G.M. 1933. Geographic variation in the big brown bat (Eptesicus fuscus). The Canadian-Field Naturalist, 47:31-32.

Álvarez, T. y O.J. Polaco. 1984. Estudio de los mamíferos capturados en La Michilía, sureste de Durango, México. Anales de la Escuela Nacional de Ciencias Biológicas, México, 28:99148.

Álvarez, T., S.T. Álvarez-Castañeda y J.C. López-Vidal. 1994. Claves para murciélagos mexicanos. Centro de Investigaciones Biológicas del Noroeste, S. C., La Paz, Baja California Sur, México.

Bogan, M.A. 1999. Familia Vespertilionidae. Pp. 139-181, en: Mamíferos del noroeste de México (S. T. Alvarez-Castañeda y J. L. Patton, eds.). Centro de Investigaciones Biológicas del Noroeste, S. C., La Paz, Baja California Sur, México.

Burnett, C.D. 1983. Geographic and secondary sexual variation in the morphology of Eptesicus fuscus. Annals of the Carnegie Museum, 52:139-161.

Ceballos, G. y G. Oliva (coords.). 2005. Los mamíferos silvestres de México Comisión Nacional para el Conocimiento y Uso de la Biodiversidad y Fondo de Cultura Económica, México, D.F.

Ceballos, G., J. Arroyo-Cabrales, R.A. Medellín y Y. Domínguez-Castellanos. 2005. Lista actualizada de los Mamíferos de México. Revista Mexicana de Mastozoología, 9:21-71.

Davis, W.B. 1965. Review of the Eptesicus brasiliensis complex in Middle America with description of a new subspecies from Costa Rica. Journal of Mammalogy, 46:229-240.

Dolan, P.G. 1989. Systematics of Middle American mastiff bats of the genus Molossus. Special Publications of The Museum, Texas Tech University, 29:1-71.

Elizalde-Arellano, C., E. Uría-Galicia y J.C. López-Vidal. 2004. Estructura anatómica e histológica de la lengua del murciélago frugívoro Centurio senex (Chiroptera: Phyllostomidae). Acta Zoológica Mexicana (n. s.), 20:31-37.

Engels, W.L. 1936. Distribution of races of the brown bat (Eptesicus) in western North America. The American Midland Naturalist, 17:653-660.

Engstrom, M.D. y D.E. Wilson. 1981. Systematics of Antrozous dubiaquercus (Chiroptera: Vespertilionidae), with comments on the status of Bauerus Van Gelder. Annals of the Carnegie Museum, 50:371-383.

Flores Villela, O. y P. Gerez. 1994. Biodiversidad y conservación en México: vertebrados, vegetación y uso del suelo. Comisión Nacional para el Conocimiento y Uso de la Biodiversidad y Universidad Nacional Autónoma de México. 
Gardner, A.L. 1966. A new subspecies of the Aztec mastiff bat, Molossus aztecus Saussure, 1860 from southern Mexico. Los Angeles County Museum, Contributions in Science, 111:1-5.

Gardner, A.L. (editor). 2008. Mammals of South America. Volume 1 Marsupials, Xenarthrans, Shrews, and Bats. The University of Chicago Press, Chicago.

Genoways, H.H. y J.K. Jones, Jr. 1969. Taxonomic status of certain long-eared bats (genus Myotis) from the southwestern United States and Mexico. The Southwestern Naturalist, 14:1-13.

Goldman, E.A. 1951. Biological Investigations in Mexico. Smithsonian Miscellaneous Collections, 115:1-476.

Guerrero, S. y F.A. Cervantes. 2003. Lista comentada de los mamíferos terrestres del estado de Jalisco, México. Acta Zoológica Mexicana (n.s.), 89:93-110.

Hall, E.R. 1981. The Mammals of North America. Vol. I. John Wiley \& Sons, Inc., New York. Handley, C.O., Jr. 1959. A revision of the American bats of the genera Euderma and Plecotus. Proceedings of the Unites States National Museum, 110:95-246.

Hoffmeister, D.F. 1986. Mammals of Arizona. The University of Arizona Press, Tucson.

Jones, J.K., Jr. 1964. Bats from western and southern Mexico. Transactions of the Kansas Academy of Sciences, 67:509-516.

Jones, J.K., Jr., J. Arroyo-Cabrales y R.D. Owen. 1988. Revised checklist of bats (Chiroptera) of Mexico and Central America. Occasional Papers of the Museum, Texas Tech University, 120:1-34.

Jones, J.K., Jr., J.R. Choate y A. Cadena. 1972. Mammals from the Mexican state of Sinaloa. II. Chiroptera. Occasional Papers, Museum of Natural History, The University of Kansas, 6:1-29.

Juárez G., J., T. Jiménez A. y D. Navarro L. 1988. Additional records of Bauerus dubiaquercus (Chiroptera: Vespertilionidae) in Mexico. The Southwestern Naturalist, 33:385.

Koopman, K.F. 1989. A review and analysis of the bats of the West Indies. Pp. 635-644, en: Biogeography of West Indies: past, present, and future (C.A. Wood, ed.). Sandhill Crane Press, Gainesville, Florida, EUA.

Koopman, K.F. 1993. Order Chiroptera. Pp. 137-241, en: Mammal species of the world, a taxonomic and geographic reference $2^{\mathrm{a}}$ edición (D.E. Wilson y D.M. Reeder, eds.). Smithsonian Institution Press, Washington, D.C.

Koopman, K.F. 1994. Handbuch der Zoologie, Vol. VIII Mammalia. Part 60: Chiroptera: Systematics. Walter de Gruyter, Berlin.

León P., L. y E.V. Romo. 1991. Catálogo de la Colección de Mamíferos del Museo de Zoología de la Facultad de Ciencias, UNAM. Serie Catálogos del Museo de Zoología "Alfonso L. Herrera", 2:1-68.

López-González, C. 2005. Murciélagos de Paraguay. BIOSFERA, Publicaciones del Comité Español del Programa MaB y de la Red IberoMaB de la UNESCO, Madrid.

López-Wilchis, R. y J. López Jardines. 1998. Los mamíferos de México depositados en colecciones de Estados Unidos y Canadá. Vol. 1. Universidad Autónoma Metropolitana, Unidad Iztapalapa, México. 
López-Wilchis, R. y J. López Jardines. 1999. Los mamíferos de México depositados en colecciones de Estados Unidos y Canadá. Vol. 2. Universidad Autónoma Metropolitana, Unidad Iztapalapa, México.

López-Wilchis, R. y J. López Jardines. 2000. Los mamíferos de México depositados en colecciones de Estados Unidos y Canadá. Vol. 3. Universidad Autónoma Metropolitana, Unidad Iztapalapa, México.

Martin, C.O. y D.J. Schmidly. 1982. Taxonomic review of the pallid bat, Antrozous pallidus (Le Conte, 1856). Special Publication of the Museum, Texas Tech University, 18:1-48.

Medellín, R.A., H.T. Arita y O. Sánchez H. 2009. Identificación de los murciélagos de México. Claves de campo. Segunda edición. Asociación Mexicana de Mastozoología, A.C.

Mehrhoff, L.J. 1996. Museums, Research Collections, and the Biodiversity Challenge. Pp. 447-465, en: Biodiversity II. Understanding and Protecting Our Biological Resources (M.L. Reaka-Kudla, D.E. Wilson y E.O. Wilson, eds.). Joseph Henry Press, Washington, D.C.

Miller, G.S., Jr. 1897. Revision of the North American bats of the family Vespertilionidae. North American Fauna, 13:1-97.

Muñiz-Martínez, R., C. López-González, J. Arroyo-Cabrales y M. Ortiz Gómez. 2003. Noteworthy records of free-tailed bats (Chiroptera: Molossidae) from Durango, México. The Southwestern Naturalist, 48:138-144.

Owen, R.D. 1987. Phylogenetic analyses of the bat subfamily Stenodermatinae (Mammalia, Chiroptera). Special Publication of the Museum, Texas Tech University, 26:1-65.

Patten, M.A. 2004. Correlates of species richness in North American bat families. Journal of Biogeography, 31:975-985.

Polaco, O.J., J. Arroyo-Cabrales y J.K. Jones, Jr. 1992. Noteworthy records of some bats from México, The Texas Journal of Science, 44:331-338.

Ramírez-Pulido, J., A. Castro-Campillo, J. Arroyo-Cabrales y F.A. Cervantes. 1996. Lista taxonómica de los mamíferos terrestres de México. Occasional Papers of the Museum, Texas Tech University, 158:1-62.

Santos Moreno, J.A. 1994. Evaluación del uso de medidas externas estándar en los análisis morfométricos de mamíferos. Anales del Instituto de Biología, Universidad Nacional Autónoma de México, Serie Zoología, 65:275-285.

Tumlison, R. 1991. Bats of the genus Plecotus in Mexico: discrimination and distribution. Occasional Papers of the Museum, Texas Tech University, 140:1-19.

Watkins, L.C., J.K. Jones, Jr. y H.H. Genoways. 1972. Bats of Jalisco, México. Special Publication of the Museum, Texas Tech University, 1:1-44.

Williams, D.F. 1978. Taxonomic and karyologic comments on small brown bats, genus Eptesicus, from South America. Annals of the Carnegie Museum, 47:361-383.

Willig, M.R. y K.W. Selcer. 1989. Bat species density gradients in the New World: a statistical assessment. Journal of Biogeography, 16:189-195.

Wilson, D.E. 1991. Mammals of the Tres Marias Islands. Pp. 214-250, en: Contributions to Mammalogy in honor of Karl F. Koopman (T.A. Griffiths y D. Klingeners, eds.). Bulletin of the American Museum of Natural History, 206:1-432. 
Apéndice I. Medidas somáticas y craneales de los ejemplares de las especies de murciélagos (Mammalia: Chiroptera) registradas para el estado de Nayarit, México.

\begin{tabular}{|c|c|c|c|c|c|c|}
\hline $\begin{array}{l}\text { GENERO } \\
\text { ESPECIE }\end{array}$ & $\begin{array}{l}\text { Centurio } \\
\text { senex }\end{array}$ & $\begin{array}{c}\text { Choeronycteris } \\
\text { mexicana }\end{array}$ & $\begin{array}{c}\text { Gyphonycteris } \\
\text { syluestris }\end{array}$ & $\begin{array}{c}\text { Antrozous } \\
\text { paidius }\end{array}$ & $\begin{array}{c}\text { Bawerus } \\
\text { dubianercus }\end{array}$ & $\begin{array}{c}\text { Corynorhinus } \\
\text { townsendï }\end{array}$ \\
\hline & & & $\mathrm{F}$ & $\mathrm{m}$ & & \\
\hline$S E \times O$ & MIF & MF & $\mathrm{F}$ & $M$ & $M$ & $\mathrm{~F}$ \\
\hline$N$ & $28(c-22)$ & 6 & $\stackrel{1}{1}$ & $\begin{array}{l}1 \\
1\end{array}$ & 1 & \\
\hline ТОТА & $\begin{array}{l}59.39 \\
55-65\end{array}$ & $\begin{array}{c}79.2 \\
78--81\end{array}$ & $\begin{array}{l}70 \\
72\end{array}$ & $\begin{array}{l}122 \\
117\end{array}$ & 112 & 106 \\
\hline COLA & $\begin{array}{l}0 \\
0\end{array}$ & $\stackrel{9}{5}-10$ & $\begin{array}{l}12 \\
13\end{array}$ & $\begin{array}{l}52 \\
41\end{array}$ & 39 & 46 \\
\hline РАTA & $\begin{array}{c}13.18 \\
11-17\end{array}$ & $12--14^{13} 16-18$ & $\begin{array}{c}12 \\
8\end{array}$ & $\begin{array}{l}13 \\
14\end{array}$ & 12 & 10 \\
\hline OREJA & $\begin{array}{c}16.18 \\
14-17\end{array}$ & 17 & $\begin{array}{l}22 \\
21\end{array}$ & $\begin{array}{l}30 \\
31\end{array}$ & 23 & 38 \\
\hline PESO & $\begin{array}{l}19.04 \\
15-25\end{array}$ & $\begin{array}{c}16 \\
15--18\end{array}$ & $\begin{array}{c}9.9 \\
11.8\end{array}$ & $\begin{array}{l}19 \\
18\end{array}$ & 16 & \\
\hline $\mathrm{AB}$ & $\begin{array}{c}42.07 \\
40.24-44.94\end{array}$ & $\begin{array}{c}43.31 \\
41.55-44.67\end{array}$ & $\begin{array}{c}43.51 \\
43\end{array}$ & $\begin{array}{l}56.39 \\
53.97\end{array}$ & 50.73 & 42.68 \\
\hline TRA & $\begin{array}{l}4.67 \\
4-6\end{array}$ & $\begin{array}{c}8 \\
7--8\end{array}$ & 9.5 & 15 & & 18 \\
\hline GLS & $\begin{array}{c}18.43 \\
17.46-19.11\end{array}$ & $\begin{array}{c}29.94 \\
29.52-30.15\end{array}$ & $\begin{array}{l}21.23 \\
20.68\end{array}$ & $\begin{array}{l}21.26 \\
22.46\end{array}$ & 20.69 & \\
\hline СОВA & $14.36-15.28$ & $\begin{array}{c}28.84 \\
28.46-29.03\end{array}$ & $\begin{array}{c}19.25 \\
19.5\end{array}$ & $\begin{array}{l}18.70 \\
19.88\end{array}$ & 17.95 & \\
\hline $\mathrm{cocl}$ & $\begin{array}{c}15.21 \\
14.53-15.66\end{array}$ & & & $\begin{array}{l}19.37 \\
20.27\end{array}$ & 18.25 & \\
\hline ZYGO & $\begin{array}{c}14.98 \\
14.47-15.43\end{array}$ & & $\begin{array}{l}10.26 \\
10.63\end{array}$ & 13.29 & 12.94 & \\
\hline MAST & $\begin{array}{c}11.84 \\
11.57-12.16\end{array}$ & $\begin{array}{c}10.17 \\
9.88-10.46\end{array}$ & $\begin{array}{l}9.62 \\
9.72\end{array}$ & $\begin{array}{c}9.84 \\
10.14\end{array}$ & 9.87 & \\
\hline OREI & 5.89 & $\begin{array}{c}4.14 \\
3.86-4.16\end{array}$ & $\begin{array}{l}4.83 \\
4.87\end{array}$ & $\begin{array}{c}4 \\
4.01\end{array}$ & 4.07 & \\
\hline BRAB & $\begin{array}{c}10.06 \\
9.31-11.09\end{array}$ & $\begin{array}{c}9.87 \\
9.65-10.17\end{array}$ & $\begin{array}{l}8.55 \\
9.02\end{array}$ & $\begin{array}{l}8.58 \\
8.80\end{array}$ & 7.95 & \\
\hline ERAH & $\begin{array}{c}11.55 \\
10.87-12.00\end{array}$ & $\begin{array}{c}9.36 \\
9.06-9.52\end{array}$ & & $\begin{array}{l}8.88 \\
8.74\end{array}$ & 9.35 & \\
\hline$P A L A$ & $\begin{array}{c}3.83 \\
3.43-4.19\end{array}$ & $\begin{array}{c}18.18 \\
17.64-18.65\end{array}$ & 9.09 & $\begin{array}{l}9.33 \\
9.69\end{array}$ & 7.54 & \\
\hline POST & $\begin{array}{c}8.73 \\
8.39-9.20\end{array}$ & $\begin{array}{c}822 \\
7.87-8.52\end{array}$ & 705 & $\begin{array}{l}7.31 \\
7.66\end{array}$ & 6.50 & \\
\hline MAXIL & $\begin{array}{c}5.03 \\
4.57-5.27\end{array}$ & $\begin{array}{c}11.38 \\
11.07-11.7\end{array}$ & $\begin{array}{l}8.16 \\
8.52\end{array}$ & $\begin{array}{l}7.35 \\
7.44\end{array}$ & 6.75 & \\
\hline WM12 & $\begin{array}{c}6.48 \\
6.26-6.76\end{array}$ & $\begin{array}{c}3.58 \\
3.43-3.67\end{array}$ & 338 & $\begin{array}{l}3.37 \\
3.72\end{array}$ & 3.26 & \\
\hline DENT & $\begin{array}{c}10.68 \\
9.97-11.10\end{array}$ & $\begin{array}{c}21.6 \\
21.31-21.91\end{array}$ & 13.84 & $\begin{array}{c}14.12 \\
15\end{array}$ & 13.80 & \\
\hline ТОOT & $\begin{array}{c}5.67 \\
5.46-5.87\end{array}$ & $\begin{array}{c}11.89 \\
11.58-12.01\end{array}$ & 9.15 & $\begin{array}{l}7.99 \\
8.20\end{array}$ & 7.63 & \\
\hline COCA & $\begin{array}{c}10.10 \\
9.74-10.46\end{array}$ & $\begin{array}{c}20.87 \\
20.44-21.31\end{array}$ & & $\begin{array}{l}13.71 \\
14.34\end{array}$ & 13.34 & \\
\hline como & $\begin{array}{c}4.35 \\
3.95-4.74\end{array}$ & $\begin{array}{c}8.8 \\
8.42-9\end{array}$ & & $\begin{array}{l}5.36 \\
5.68\end{array}$ & 5.09 & \\
\hline CORO & $\begin{array}{c}4.18 \\
3.73-4.37\end{array}$ & $\begin{array}{c}4.7 \\
4.33-4.97\end{array}$ & & $\begin{array}{l}6.36 \\
6.89\end{array}$ & 6.29 & \\
\hline THICK & $\begin{array}{c}2.59 \\
2.29-2.83\end{array}$ & $\begin{array}{c}1.73 \\
1.59-1.87\end{array}$ & & $\begin{array}{l}1.95 \\
2.06\end{array}$ & 2.76 & \\
\hline COND & $\begin{array}{c}1.97 \\
1.75-2.10\end{array}$ & $\begin{array}{c}1.7 \\
1.47-1.98\end{array}$ & & $\begin{array}{l}2.55 \\
2.76\end{array}$ & 2.49 & \\
\hline
\end{tabular}


Apéndice I. Continuación....

\begin{tabular}{|c|c|c|c|c|c|}
\hline $\begin{array}{l}\text { GENERO } \\
\text { ESPECIE }\end{array}$ & $\begin{array}{c}\text { Eptesicus } \\
\text { fwninais }\end{array}$ & $\begin{array}{c}\text { Entesicus } \\
\text { fuscus }\end{array}$ & $\begin{array}{c}\text { Eptesicus } \\
\text { fuscus }\end{array}$ & $\begin{array}{l}\text { Myotis } \\
\text { ankicus }\end{array}$ & $\begin{array}{c}\text { Myotis } \\
\text { byysenowes }\end{array}$ \\
\hline$S E \times O$ & $\mathrm{~F}$ & $F$ & ro1 & $\mathrm{m} / \mathrm{F}$ & $\mathrm{M} / \mathrm{F}$ \\
\hline N & $\begin{array}{l}1 \\
1\end{array}$ & 5 & 7 & 14 & 8 \\
\hline TOTA. & $\begin{array}{l}92 \\
99\end{array}$ & $\begin{array}{c}113.20 \\
105-116\end{array}$ & $\begin{array}{c}109 \\
97-125\end{array}$ & $\begin{array}{l}88.64 \\
78-95\end{array}$ & $\begin{array}{l}85.75 \\
84-88\end{array}$ \\
\hline COLA. & $\begin{array}{l}33 \\
34\end{array}$ & $\begin{array}{l}3820 \\
33-44\end{array}$ & $\begin{array}{l}37 \cdot 71 \\
31-45\end{array}$ & $\begin{array}{l}37 \cdot 36 \\
31-44\end{array}$ & $\begin{array}{l}32.63 \\
29-35\end{array}$ \\
\hline P.ATA. & $\begin{array}{l}7 \\
9\end{array}$ & $g_{-11}^{10}$ & $\begin{array}{l}9.8 \mathrm{~B} \\
7-12\end{array}$ & $\begin{array}{c}9.57 \\
8--10\end{array}$ & $\begin{array}{c}9.63 \\
9--10\end{array}$ \\
\hline OREJA. & $\begin{array}{l}14 \\
15\end{array}$ & $\begin{array}{l}1680 \\
15-19\end{array}$ & $\begin{array}{c}16 \\
12-18\end{array}$ & $\frac{20}{17-23}$ & $\begin{array}{l}18.88 \\
18-20\end{array}$ \\
\hline PESO & $\begin{array}{c}7 \\
11.2\end{array}$ & $\begin{array}{c}17.80 \\
14-20.4\end{array}$ & $\begin{array}{c}14.80 \\
10-18.1\end{array}$ & $\begin{array}{c}7.1 \\
4-20.4\end{array}$ & $\begin{array}{l}6.40 \\
5--8\end{array}$ \\
\hline 䖧 & $\begin{array}{l}39.45 \\
39.85\end{array}$ & $\begin{array}{c}47.73 \\
46.89-48.97\end{array}$ & $\begin{array}{c}46.82 \\
44.85-51.65\end{array}$ & $\frac{37.04}{34.60-38.11}$ & $\begin{array}{c}41.07 \\
39.84-42.45\end{array}$ \\
\hline TRA. & 7 & $\begin{array}{l}8.50 \\
8--9\end{array}$ & $\begin{array}{l}10.25 \\
9-13\end{array}$ & $\begin{array}{c}11.91 \\
11--14\end{array}$ & $\begin{array}{l}10.50 \\
5--12\end{array}$ \\
\hline GLS & $\begin{array}{c}15.4 \\
16\end{array}$ & $\begin{array}{c}1980 \\
19.13-21.17\end{array}$ & $\begin{array}{c}19.11 \\
18.49-20.08\end{array}$ & $\begin{array}{c}16.53 \\
15.81-16.91\end{array}$ & $\begin{array}{c}16.58 \\
16.14-16.85\end{array}$ \\
\hline $\operatorname{co\theta } A$ & $\begin{array}{l}14.35 \\
14.97\end{array}$ & $\begin{array}{c}1823 \\
17.39-19.34\end{array}$ & $17.22-18.62$ & $\begin{array}{l}15.35 \\
14.80-15.70\end{array}$ & $\begin{array}{c}15.27 \\
14.90-15.65\end{array}$ \\
\hline $\mathrm{coc}$ & $\begin{array}{l}1456 \\
1522\end{array}$ & $\begin{array}{c}18.54 \\
17.92-19.54\end{array}$ & $\begin{array}{c}18.06 \\
17.45-18.93\end{array}$ & $\begin{array}{c}15.58 \\
15.01-16.02\end{array}$ & $\begin{array}{c}15.50 \\
15.13-15.90\end{array}$ \\
\hline Zroo & $\begin{array}{c}9.94 \\
10.34\end{array}$ & $\begin{array}{c}1288 \\
12.30-13.65\end{array}$ & $\begin{array}{c}12.60 \\
12.26-13.33\end{array}$ & $\begin{array}{c}8.99 \\
9.51-10.0\end{array}$ & $\begin{array}{c}10.16 \\
9.88-10.85\end{array}$ \\
\hline$M A S T$ & $\begin{array}{l}8.25 \\
8.34\end{array}$ & $\begin{array}{c}9.92 \\
9.35-10.43\end{array}$ & $\begin{array}{c}9.77 \\
9.51-10.16\end{array}$ & $\frac{8.27}{8.03-8.55}$ & $\begin{array}{c}8.07 \\
7.89-8.39\end{array}$ \\
\hline OR日I & $\begin{array}{l}4.05 \\
4.11\end{array}$ & $\begin{array}{c}4.20 \\
4.11-4.41\end{array}$ & $\begin{array}{c}4.24 \\
3.994 .42\end{array}$ & $\begin{array}{c}3.9 \\
3.72-4.02\end{array}$ & $\begin{array}{c}4.06 \\
3.90-4.29\end{array}$ \\
\hline 日R:А日 & 7.45 & $\begin{array}{c}8.84 \\
8.19-9.05\end{array}$ & $\begin{array}{c}8.50 \\
8.09-8.87\end{array}$ & $\begin{array}{c}7.55 \\
7.23-8.03\end{array}$ & $\frac{7.82}{7.71-7.92}$ \\
\hline BR:AH & $\frac{7.22}{7.41}$ & $\begin{array}{c}7.77 \\
7.21-8.25\end{array}$ & $\begin{array}{c}7.59 \\
7.30-8.20\end{array}$ & $\frac{7.07}{6.74-7.45}$ & $\begin{array}{c}6.93 \\
6.62-7.24\end{array}$ \\
\hline$P, A L A$. & $\begin{array}{l}6.75 \\
5.96\end{array}$ & $\begin{array}{c}8.25 \\
7.44-9.10\end{array}$ & $\begin{array}{c}8.19 \\
7.28-9.45\end{array}$ & $\begin{array}{c}8.28 \\
7.25-8.76\end{array}$ & $\begin{array}{c}8.41 \\
8.12-8.64\end{array}$ \\
\hline POST & $\begin{array}{l}5.94 \\
6.19\end{array}$ & $\begin{array}{c}7.15 \\
6.85-7.35\end{array}$ & $\begin{array}{c}7.04 \\
6.62-7.47\end{array}$ & $\frac{5.07}{4.74-5.48}$ & $\begin{array}{c}5.02 \\
4.83-5.22\end{array}$ \\
\hline$M 1, \triangle \times 1 L$ & $\begin{array}{l}5.54 \\
5.71\end{array}$ & $\begin{array}{c}7.06 \\
6.92-7.34\end{array}$ & $\begin{array}{c}6.88 \\
6.54-7.32\end{array}$ & $\frac{6.36}{5.84-6.55}$ & $\frac{6.21}{6.01-6.54}$ \\
\hline viruz & $\begin{array}{l}2.99 \\
3.07\end{array}$ & $\begin{array}{c}3.78 \\
3.44-3.99\end{array}$ & $\begin{array}{c}3.57 \\
3.27-3.94\end{array}$ & $\frac{2.77}{2.66-2.97}$ & $\begin{array}{c}3.01 \\
2.74-3.25\end{array}$ \\
\hline DENT & $\begin{array}{l}11.19 \\
11.54\end{array}$ & $\begin{array}{c}1426 \\
13.54-15.36\end{array}$ & $\begin{array}{c}13.91 \\
13.52-14.51\end{array}$ & $\begin{array}{c}11.76 \\
11.32-12.22\end{array}$ & $\begin{array}{c}11.58 \\
11.48-11.87\end{array}$ \\
\hline TOOT & $\begin{array}{l}5.83 \\
6.08\end{array}$ & $\begin{array}{c}7.80 \\
7.44-8.38\end{array}$ & $\begin{array}{c}7.65 \\
7.30-8.03\end{array}$ & $\begin{array}{c}6.68 \\
6.36-6.91\end{array}$ & $\begin{array}{c}6.58 \\
6.34-6.80\end{array}$ \\
\hline $\operatorname{coc} A$ & $\begin{array}{l}1025 \\
10.34\end{array}$ & $\begin{array}{c}1326 \\
12.85-14.13\end{array}$ & $12.71-13.36$ & $\begin{array}{c}10.64 \\
10.14-10.99\end{array}$ & $10.20 .47-10.72$ \\
\hline como & $\begin{array}{l}4.42 \\
4.06\end{array}$ & $\begin{array}{c}5.51 \\
5.21-5.95\end{array}$ & $\begin{array}{c}5.37 \\
5.04-5.69\end{array}$ & $\begin{array}{c}3.92 \\
3.69-4.14\end{array}$ & $\begin{array}{c}3.93 \\
3.79-4.14\end{array}$ \\
\hline CORO & $\begin{array}{l}4.20 \\
1.98\end{array}$ & $\begin{array}{c}5.75 \\
5.55-5.91\end{array}$ & $\begin{array}{c}5.59 \\
5.33-5.99\end{array}$ & $\begin{array}{c}4.18 \\
3.83-4.44\end{array}$ & $\begin{array}{c}4.09 \\
3.89-4.41\end{array}$ \\
\hline$T H I C K$ & $\begin{array}{l}1.58 \\
1.30\end{array}$ & $\begin{array}{c}2.29 \\
1.94-2.53\end{array}$ & $\begin{array}{c}2.35 \\
1.74-3.00\end{array}$ & $\begin{array}{c}1.31 \\
1.15-1.78\end{array}$ & $\begin{array}{c}1.27 \\
1.12-1.53\end{array}$ \\
\hline COND & $\begin{array}{l}1.60 \\
1.62\end{array}$ & $\begin{array}{c}2.12 \\
2.04-2.32\end{array}$ & $\begin{array}{c}2.05 \\
1.89-2.28\end{array}$ & $\begin{array}{c}1.57 \\
1.41-1.68\end{array}$ & $\begin{array}{c}1.67 \\
1.55-1.77\end{array}$ \\
\hline
\end{tabular}


Apéndice I. Continuación....

\begin{tabular}{|c|c|c|c|c|c|}
\hline $\begin{array}{l}\text { GENERO } \\
\text { ESPECIE }\end{array}$ & $\begin{array}{l}\text { Myotis } \\
\text { venter }\end{array}$ & $\begin{array}{c}\text { Myotis } \\
\text { yumanensis }\end{array}$ & $\begin{array}{c}\text { Parastrems } \\
\text { nesperus }\end{array}$ & $\begin{array}{c}\text { Parastrems } \\
\text { hesperws }\end{array}$ & $\begin{array}{c}\text { Rhogeessa } \\
\text { gracilis }\end{array}$ \\
\hline$S E \times O$ & F & $\mathrm{M}$ & $\begin{array}{l}\mathrm{F} \\
\mathrm{F}\end{array}$ & $\mathrm{m}$ & $\mathrm{M}$ \\
\hline N & 1 & $\begin{array}{l}1 \\
1\end{array}$ & $\begin{array}{l}1 \\
1\end{array}$ & 1 & 1 \\
\hline TOTA & 101 & $\begin{array}{l}79 \\
70\end{array}$ & $\begin{array}{l}65 \\
71\end{array}$ & 69 & 84 \\
\hline COLA. & 43 & $\begin{array}{l}30 \\
27\end{array}$ & $\begin{array}{l}26 \\
25\end{array}$ & 25 & 40 \\
\hline PAT A. & 10 & $\frac{9}{7}$ & $\frac{6}{7}$ & 6 & 6 \\
\hline OREJA. & 19 & $\begin{array}{l}13 \\
14\end{array}$ & $\begin{array}{l}12 \\
12\end{array}$ & 11 & 19 \\
\hline PESO & 12 & $\begin{array}{l}4 \\
4\end{array}$ & $\begin{array}{l}3 \\
3\end{array}$ & 27.4 & 4 \\
\hline $\mathrm{AB}$ & 42.40 & $\begin{array}{l}32.84 \\
31.53\end{array}$ & $\frac{28.52}{29.88}$ & 27.40 & 33.56 \\
\hline TRA. & 9 & & 6 & 6 & 11 \\
\hline GLS & 16.41 & $\begin{array}{l}13.65 \\
12.98\end{array}$ & $\begin{array}{c}11.35 \\
11.7\end{array}$ & 11.37 & 13.77 \\
\hline COEA & 15.56 & $\begin{array}{l}12.51 \\
11.89\end{array}$ & $\begin{array}{c}10.8 \\
11.04\end{array}$ & 10.55 & 12.71 \\
\hline $\mathrm{cocl}$ & 15.97 & $\begin{array}{l}12.81 \\
12.15\end{array}$ & $\begin{array}{l}10.86 \\
10.88\end{array}$ & 10.65 & 12.85 \\
\hline ZYOOO & 10.74 & 7.54 & $\begin{array}{l}6.95 \\
7.30\end{array}$ & 6.91 & 8.45 \\
\hline$M A S T$ & 8.32 & $\begin{array}{l}7.02 \\
6.53\end{array}$ & $\begin{array}{l}5.97 \\
6.08\end{array}$ & 5.98 & 6.93 \\
\hline OR日I & 3.88 & $\begin{array}{l}3.95 \\
3.44\end{array}$ & $\begin{array}{l}2.99 \\
3.08\end{array}$ & 3.24 & 3.32 \\
\hline BRAB & 7.80 & $\begin{array}{l}6.73 \\
6.37\end{array}$ & $\begin{array}{l}5.93 \\
5.71\end{array}$ & 5.69 & 6.25 \\
\hline ERAH & 6.84 & $\begin{array}{l}6.14 \\
6.02\end{array}$ & $\begin{array}{l}5.34 \\
5.51\end{array}$ & 5.15 & 6.22 \\
\hline$P, A \perp A$ & 8.70 & $\begin{array}{l}6.13 \\
6.16\end{array}$ & $\begin{array}{l}4.2 \\
5.12\end{array}$ & 5.08 & 6.05 \\
\hline POST & 5.44 & $\begin{array}{l}4.93 \\
4.57\end{array}$ & $\begin{array}{l}4.54 \\
4.60\end{array}$ & 4.54 & 4.99 \\
\hline MAXIL & 6.49 & $\begin{array}{l}5.07 \\
4.69\end{array}$ & $\begin{array}{l}3.75 \\
3.87\end{array}$ & 3.78 & 4.64 \\
\hline wm2 & 3.45 & $\begin{array}{l}2.56 \\
2.36\end{array}$ & 2.5 & 2.27 & 2.62 \\
\hline DENT & 12.51 & $\begin{array}{l}9.41 \\
9.00\end{array}$ & $\begin{array}{l}7.47 \\
7.65\end{array}$ & 7.47 & 9.22 \\
\hline тоOт & 6.99 & $\begin{array}{l}5.23 \\
4.91\end{array}$ & $\begin{array}{l}3.95 \\
3.96\end{array}$ & 3.82 & 5.09 \\
\hline $\operatorname{COCA}$ & 11.36 & $\begin{array}{l}8.40 \\
8.01\end{array}$ & $\begin{array}{l}6.53 \\
6.94\end{array}$ & 6.67 & 8.46 \\
\hline cOMO & 4.45 & $\begin{array}{l}3.07 \\
3.07\end{array}$ & $\begin{array}{l}2.69 \\
2.79\end{array}$ & 2.85 & 3.20 \\
\hline coro & 4.49 & $\begin{array}{l}3.15 \\
2.54\end{array}$ & $\begin{array}{l}2.14 \\
2.43\end{array}$ & 2.27 & 3.44 \\
\hline THICK & 1.29 & $\begin{array}{l}0.95 \\
0.82\end{array}$ & $\begin{array}{l}0.98 \\
1.08\end{array}$ & 0.81 & 1.22 \\
\hline COND & 1.58 & $\begin{array}{l}1.08 \\
1.17\end{array}$ & $\begin{array}{l}1.02 \\
0.95\end{array}$ & 0.98 & 1.33 \\
\hline
\end{tabular}


Apéndice I. Continuación....

\begin{tabular}{|c|c|c|c|c|c|}
\hline $\begin{array}{l}\text { GENERO } \\
\text { ESPECIE }\end{array}$ & $\begin{array}{l}\text { Eumops } \\
\text { werotis }\end{array}$ & $\begin{array}{l}\text { Movossus } \\
\text { aztecus }\end{array}$ & $\begin{array}{l}\text { Mobssus } \\
\text { sinabae }\end{array}$ & $\begin{array}{l}\text { Notinomops } \\
\text { femonosacus }\end{array}$ & $\begin{array}{l}\text { Nyctimomops } \\
\text { laticaudatus }\end{array}$ \\
\hline$S E \times O$ & $\mathrm{~F}$ & $M / F$ & $M / F$ & $M A F$ & $M \pi$ \\
\hline$N$ & 1 & $22(c-18)$ & $8(c-6)$ & 4 & 4 \\
\hline ТОТА & 167 & $\begin{array}{l}9427 \\
90-97\end{array}$ & $\begin{array}{c}124.88 \\
121-130\end{array}$ & $\begin{array}{c}110 \\
110-110\end{array}$ & $\begin{array}{c}101 \\
98-104\end{array}$ \\
\hline COLA. & 55 & $\begin{array}{l}33.73 \\
30-37\end{array}$ & $\begin{array}{l}44.25 \\
40-48\end{array}$ & $\begin{array}{l}39.25 \\
35-41\end{array}$ & $\begin{array}{c}36 \\
34-38\end{array}$ \\
\hline P.ATA. & 15 & $\begin{array}{c}11.18 \\
10-12\end{array}$ & $\begin{array}{c}12.63 \\
11--13\end{array}$ & $\begin{array}{l}10.25 \\
8-11\end{array}$ & $\begin{array}{c}9 \\
8-10\end{array}$ \\
\hline OREJA. & 44 & $\begin{array}{c}13.73 \\
13-15\end{array}$ & $\begin{array}{l}16.25 \\
16-17\end{array}$ & $\begin{array}{c}23 \\
21-25\end{array}$ & $\begin{array}{l}19.50 \\
18-20\end{array}$ \\
\hline PESO & 46 & $\begin{array}{l}1268 \\
7-16\end{array}$ & $\begin{array}{c}27 \\
23-32\end{array}$ & $\begin{array}{c}13 \\
11-15\end{array}$ & $\begin{array}{c}11.75 \\
10-13\end{array}$ \\
\hline A日 & 75.56 & $\begin{array}{c}3621 \\
3530-36.87\end{array}$ & $\begin{array}{c}48.23 \\
47.40-48.89\end{array}$ & $\begin{array}{c}45.99 \\
45.32-46.66\end{array}$ & $\begin{array}{c}42.09 \\
41.62-43.02\end{array}$ \\
\hline TRA. & & $\begin{array}{l}4.18 \\
4-5\end{array}$ & $\begin{array}{l}5.25 \\
4--7\end{array}$ & $\stackrel{6}{5-7}$ & \\
\hline GLS & 31.77 & $\begin{array}{c}17.41 \\
1683-18.24\end{array}$ & $\begin{array}{c}21.60 \\
21.13-22.54\end{array}$ & $\begin{array}{c}19.90 \\
19.54-50.24\end{array}$ & $\begin{array}{c}18.27 \\
17.73-18.83\end{array}$ \\
\hline COEA. & 29.57 & $\begin{array}{c}15.12 \\
1467-15.67\end{array}$ & $\begin{array}{c}18.80 \\
18.07-19.33\end{array}$ & $\begin{array}{c}18.17 \\
17.99-18.39\end{array}$ & $\begin{array}{c}16.74 \\
16.41-16.91\end{array}$ \\
\hline $\mathrm{cocl}$ & 30.62 & $\begin{array}{c}15.41 \\
14.92-15.89\end{array}$ & $\begin{array}{c}19.32 \\
18.69-19.95\end{array}$ & $\begin{array}{c}18.43 \\
18.10-18.82\end{array}$ & $\begin{array}{c}16.90 \\
16.37-17.37\end{array}$ \\
\hline ZYGO & 17.79 & $\begin{array}{c}10.93 \\
1060-11.45\end{array}$ & $\begin{array}{c}12.43 \\
12.03-12.74\end{array}$ & $\begin{array}{c}11.07 \\
10.59-11.57\end{array}$ & $\begin{array}{c}10.33 \\
10.07-10.63\end{array}$ \\
\hline MAST & 15.15 & $\begin{array}{c}10.42 \\
1009-10.94\end{array}$ & $\begin{array}{c}11.92 \\
11.63-12.53\end{array}$ & $\begin{array}{c}10.54 \\
10.26-10.88\end{array}$ & $\begin{array}{c}9.90 \\
9.71-10.15\end{array}$ \\
\hline OR日I & 5.02 & $\begin{array}{c}3.85 \\
3.3-4.08\end{array}$ & $\begin{array}{c}3.86 \\
3.75-4\end{array}$ & $\begin{array}{c}3.70 \\
3.53-3.91\end{array}$ & $\begin{array}{c}3.68 \\
3.58-3.85\end{array}$ \\
\hline BRAB & 13.70 & $\begin{array}{c}8.94 \\
8.68-9.16\end{array}$ & $\begin{array}{c}9.94 \\
9.81-10.32\end{array}$ & $\begin{array}{c}9.04 \\
8.95-9.30\end{array}$ & $\begin{array}{c}8.73 \\
8.59-9.01\end{array}$ \\
\hline BR $\wedge \mathrm{H}$ & 11.85 & $\begin{array}{c}7.76 \\
758-7.93\end{array}$ & $\begin{array}{c}8.91 \\
8.28-9.32\end{array}$ & $\begin{array}{c}7.39 \\
6.88-8.32\end{array}$ & $\begin{array}{c}7.44 \\
7.19-7.72\end{array}$ \\
\hline$P A \perp A$. & 12.70 & $\begin{array}{c}5.70 \\
5.43-5.98\end{array}$ & $\begin{array}{c}7.37 \\
7.09-7.74\end{array}$ & $\begin{array}{c}8.14 \\
7.77-8.37\end{array}$ & $\begin{array}{c}7.29 \\
6.92-7.48\end{array}$ \\
\hline POST & 14 & $\begin{array}{c}7.29 \\
6.96-7.70\end{array}$ & $\begin{array}{c}8.86 \\
8.51-9.28\end{array}$ & $\begin{array}{c}8.12 \\
7.96-8.28\end{array}$ & $\begin{array}{c}7.89 \\
7.53-8.18\end{array}$ \\
\hline$M A X I L$ & 11.68 & $\begin{array}{c}5.85 \\
5.67-6.15\end{array}$ & $\begin{array}{c}7.42 \\
7.24-7.66\end{array}$ & $\begin{array}{c}7.23 \\
7.08-7.37\end{array}$ & $\begin{array}{c}6.86 \\
6.29-7.94\end{array}$ \\
\hline WM2 & 5.34 & $\begin{array}{c}3.67 \\
3.46-3.87\end{array}$ & $\begin{array}{c}4.01 \\
3.91-4.13\end{array}$ & $\begin{array}{c}3.40 \\
3.33-3.46\end{array}$ & $\begin{array}{c}3.14 \\
2.95-3.28\end{array}$ \\
\hline DENT & 22.66 & $\begin{array}{c}1151 \\
10.79-11.95\end{array}$ & $\begin{array}{c}14.42 \\
13.93-14.93\end{array}$ & $\begin{array}{c}13.41 \\
12.94-13.88\end{array}$ & $\begin{array}{c}12.34 \\
11.70-12.77\end{array}$ \\
\hline TOOT & 12.83 & $\begin{array}{c}6.56 \\
6.19-6.93\end{array}$ & $\begin{array}{c}8.39 \\
8.19-8.60\end{array}$ & $\begin{array}{c}7.77 \\
7.52-8.11\end{array}$ & $\begin{array}{c}7.17 \\
6.84-7.37\end{array}$ \\
\hline COCA. & 21.95 & $\begin{array}{c}1133 \\
10.95-11.88\end{array}$ & $\begin{array}{c}14.17 \\
13.73-14.69\end{array}$ & $\begin{array}{c}12.97 \\
12.51-13.42\end{array}$ & $\begin{array}{c}11.79 \\
11.27-12.12\end{array}$ \\
\hline como & 8.87 & $\begin{array}{c}4.61 \\
428-4.90\end{array}$ & $\begin{array}{c}5.60 \\
5.28-5.96\end{array}$ & $\begin{array}{c}4.78 \\
3.86-5.29\end{array}$ & $\begin{array}{c}4.64 \\
4.40-4.85\end{array}$ \\
\hline CORO & 5.30 & $\begin{array}{c}4.31 \\
3.85-4.78\end{array}$ & $\begin{array}{c}5.45 \\
5.25-5.80\end{array}$ & $\begin{array}{c}2.94 \\
2.15-3.27\end{array}$ & $\begin{array}{c}3.35 \\
3.17-3.63\end{array}$ \\
\hline THICK & 3.15 & $\frac{2.05}{1.83-2.21}$ & $\begin{array}{c}2.79 \\
2.58-2.93\end{array}$ & $\begin{array}{c}1.88 \\
1.67-2.10\end{array}$ & $\begin{array}{c}1.84 \\
1.68-2\end{array}$ \\
\hline COND & 2.37 & $\begin{array}{c}1.45 \\
1.35-1.52\end{array}$ & $\begin{array}{c}1.91 \\
1.83-1.97\end{array}$ & $\begin{array}{c}1.37 \\
1.28-1.49\end{array}$ & $\frac{1.23}{1.16-1.32}$ \\
\hline
\end{tabular}




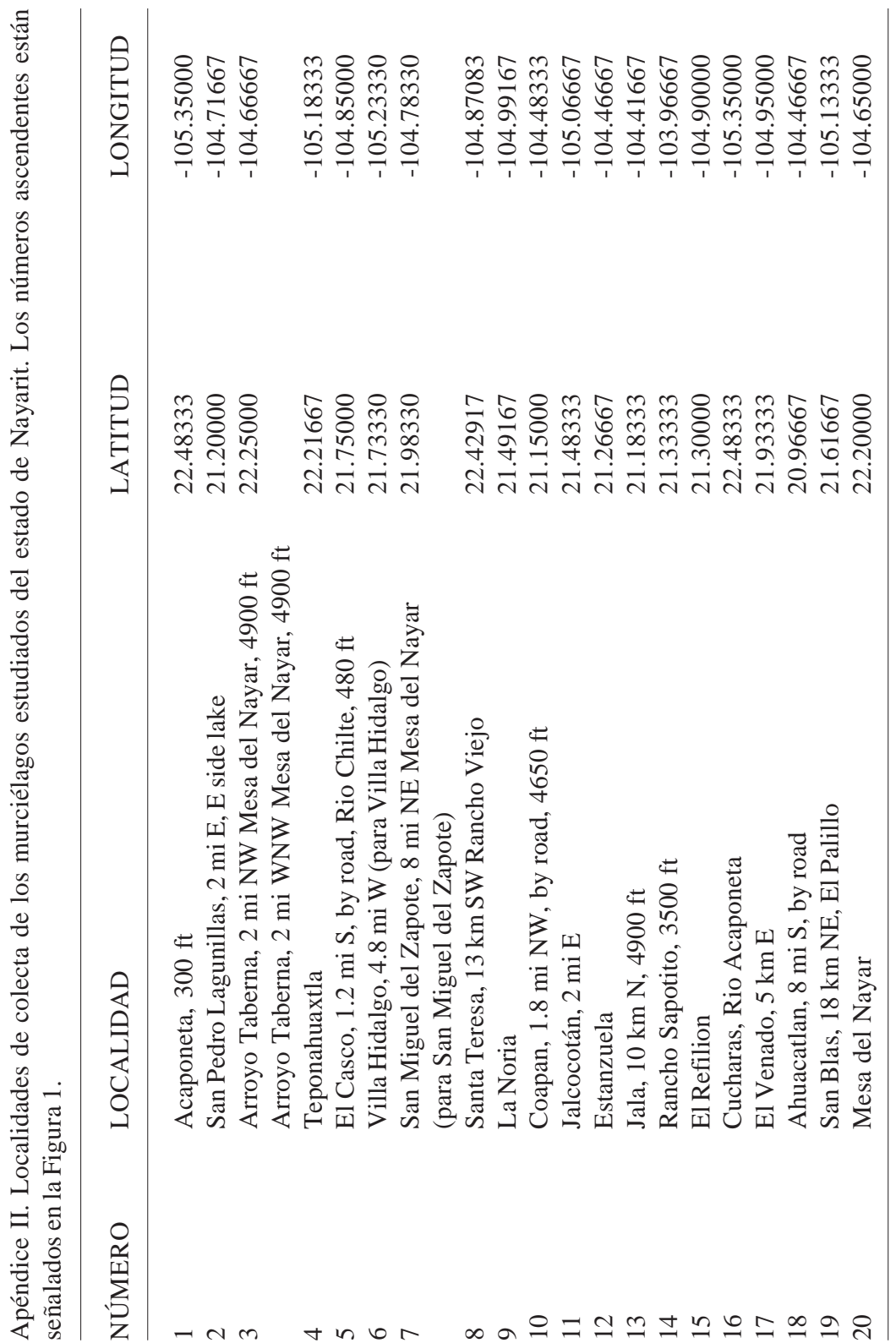


Apéndice III. Listado de los ejemplares procedentes del estado de Nayarit y en depósito en la Colección de Mamíferos del National Museum of Natural History, Washington, D. C. (USNM). Un ejemplar procedente de la Colección de Mamíferos de The University of Kansas Museum of Natural History (KU) fue también revisado.

Centurio senex

USNM 508827, 511473, 511474, 511475, 511476, 511477, 511478, 511479, 511480, 511481, 511482, 511483, 511484, 511485, 511486, 511487, 511488, 511740, 511741, 511854, 511855, 511856, 511857, 511858, 511859, 559704, 559705, 559706

Choeronycteris mexicana

USNM 91332, 508546, 511302, 511303, 511304, 511305

Glyphonycteris sylvestris

KU 96970

Antrozous pallidus

USNM 511540, 523452

Coryhorhinus townsendii

USNM 511539

Eptesicus furinalis

USNM 523441

Eptesicus fuscus

USNM 508864, 508865, 511521, 511522, 511523, 511524

Myotis auriculus

USNM 508852-508857, 511493-511497

Myotis thysanodes

USNM 508861, 511512-511518

Myotis velifer

USNM 558862

Myotis yumanensis

USNM 508863, 559757 
Apéndice III. Continuación...

Parastrellus hesperus

USNM 511519, 511520, 559768

Rhogeessa gracilis

USNM 523442

Eumops perotis

USNM 509002

Molossus aztecus

USNM 508978-508995, 510433-510436

Molossus sinaloae

USNM 508996-509001, 510437, 510438

Nyctinomops femorosaccus

USNM 510423, 510424, 511541, 511542, 559794, 559795

Nyctinomops laticaudatus

USNM 508920, 508922, 508923 